\title{
Charles Pecher et Edgar Sengier
}

\section{Tribulations de deux "héros" belges à l'aube de la seconde guerre mondiale et de l'ère nucléaire.}

Amand LUCAS

Universités de Namur et de Liège

\section{Résumé}

Je présente brièvement les carrières contrastées de deux éminentes personnalités belges, l'une scientifique, l'autre industrielle, et leurs interactions souvent problématiques avec deux gouvernements, celui de la Belgique et celui des États-Unis, à un moment tragique de l'histoire que fut le début de la seconde guerre mondiale.

\section{Introduction}

Leur nationalité belge mise à part, Pecher (1913-1941) et Sengier (1879-1963) ont apparemment peu de choses en commun : une génération les sépare, le premier est jeune médecin-chercheur tandis que le second est ingénieur chevronné, capitaine d'industrie. En outre leurs destins furent radicalement différents. Cela étant, l'un comme l'autre ont joué un rôle éminent dans l'avènement de l'ère nucléaire au début de la guerre en 1939. En février 1940, Pecher arrive à Berkeley sur la côte ouest des États-Unis. Il y participe aux recherches sur les applications médicales des radioéléments produits par le cyclotron, l'accélérateur de particules inventé par Ernest Lawrence en 1932. Il démontrera, entre autres grandes découvertes, les propriétés thérapeutiques du strontium-89 en usage aujourd'hui. A la fin de 1939, Sengier sous la menace allemande, s'est réfugié à New York pour diriger les affaires commerciales et financières de l'Union Minière du Haut Katanga (UMHK) sur laquelle il détient la haute main. En particulier, il y organisera le stockage et la vente du riche minerai d'uranium katangais, un matériau devenu éminemment convoité après la découverte de la fission de l'uranium en 1938-39 par Hahn et Strassmann à Berlin.

Par la suite, les destins de nos deux "héros" divergeront dramatiquement. Pour le premier, le prestige et le crédit que lui confèrent ses découvertes précoces aux États-Unis ne lui épargneront pas une mort tragique et mystérieuse au Canada en 1941. Son œuvre restera étouffée par le secret atomique pendant un demi-siècle. Tandis que le second jouira d'une grande reconnaissance, bien que maintenue secrète elle aussi, pour la fourniture de l'uranium utilisé pour le bombardement atomique du Japon.

Tous deux furent confrontés au gouvernement belge en exil à Londres. Celui-ci est resté complètement ignorant de l'énorme importance des activités de ces deux éminents compatriotes depuis le début de la guerre. En 1941, Pecher reçut l'ordre de rejoindre l'armée belge en reconstruction au Canada et, en même temps, fut sommé par les Américains de ne pas quitter les États-Unis. Ces injonctions contradictoires provoquèrent chez le génial 
médecin-chercheur un immense déchirement personnel à l'origine de son décès. Son œuvre, largement tombée dans les oubliettes du secret nucléaire, y serait restée sans les efforts soutenus de sa fille, Evelyne Cerf-Pecher, pour raviver sa mémoire [1]. Quant à Sengier, il fut lui aussi appelé à rendre des comptes devant ce même gouvernement belge en exil. La rencontre eut lieu en 1944, à la veille de la libération de la Belgique. Elle eut un dénouement plus heureux : un accord tenu secret lui aussi sur la fourniture lucrative et exclusive aux ÉtatsUnis et au Royaume Uni des réserves d'uranium du Congo Belge. Grâce à Sengier la Belgique pourra bénéficier de cet accord, à plusieurs titres, durant les décennies post conflit.

\section{Charles Pecher.}

L'histoire de la courte et tragique vie de Charles Pecher est racontée en détail pas sa fille Evelyne dans ses publications [1]. Dans le présent article, j'en ai extrait seulement quelques informations factuelles. Un autre exposé sur Pecher s'inspirant de la même source est donné par André Jaumotte et Frank Deconinck dans une édition récente de La Nouvelle Biographie Nationale [2].

Charles Pecher est né à Anvers le 26 novembre 1913. Il accomplit de brillantes études secondaires et universitaires. Il obtient son diplôme de docteur en médecine à l'Université Libre de Bruxelles en juillet 1939. Il épouse Jacqueline Van Halteren en août 1939. Le couple s'embarque aussitôt pour les Etats-Unis grâce à un fellowship que Pecher obtient de la BAEF (Belgian American Educational Foundation). Pecher commence par augmenter ses connaissances de physique et de chimie en suivant un semestre de cours à Harvard, MA. Puis, il souhaite faire des recherches dans le domaine nouveau des applications de la radioactivité artificielle (découverte en 1934 par Frédéric et Irène Joliot-Curie) en médecine et en biologie. En février 1940, il rejoint le Radiation Laboratory d'Ernest Lawrence à Berkeley, CA, le laboratoire pionnier sur ces questions à l'époque.

Pecher y déploie une créativité peu ordinaire. En un peu plus d'un an, il produit une dizaine de publications, deux brevets d'invention (cédés au bénéfice d'une société américaine pour le développement des sciences) et démontre deux utilisations médicales nouvelles de radioisotopes qui continuent d'être exploitées de nos jours. La première est le "bone scan", désignée aujourd'hui sous le nom de scintigraphie osseuse. Il s'agit d'une méthode diagnostique d'autoradiographie par laquelle le squelette est révélé par les radiations émises par un composé chimique se fixant préférentiellement sur les os et comportant un radioélément (le radio-calcium ou le radio-strontium). De nos jours, la scintigraphie osseuse est couramment réalisée au moyen du technétium- $99 \mathrm{~m}\left({ }^{99 \mathrm{~m}} \mathrm{Tc}\right)$, un isotope métastable $(\mathrm{m}) \mathrm{de}$ 6 heures de demi-vie, émetteur de rayonnement $\gamma(141 \mathrm{keV})$ détectable à l'extérieur de l'organisme. Ce radioélément est préalablement fixé sur une molécule ayant une affinité spécifique pour les os. La seconde utilisation, plus importante encore, est la démonstration par Pecher des propriétés thérapeutiques du radio-isotope strontium-89 $\left({ }^{89} \mathrm{Sr}\right)$ [3], choisi par lui comme analogue du calcium, pour le traitement des lésions osseuses. Pecher préparait cet isotope en irradiant $\mathrm{du}{ }^{88} \mathrm{Sr}$ naturel (stable) par un faisceau de deutérium $\left({ }^{2} \mathrm{H}\right.$ ou d) accéléré dans le cyclotron de Berkeley [3]. La réaction de production est ${ }^{88} \mathrm{Sr}(\mathrm{d}, \mathrm{p}){ }^{89} \mathrm{Sr}$ (notation compacte de la réaction nucléaire ${ }^{88} \mathrm{Sr}+{ }^{2} \mathrm{H} \rightarrow{ }^{89} \mathrm{Sr}+{ }^{1} \mathrm{H}$ ) et la réaction de 
décroissance ${ }^{89} \mathrm{Sr} \rightarrow{ }^{89} \mathrm{Y}+\beta^{-}$. La demi-vie du ${ }^{89} \mathrm{Sr}$ est de 50,5 jours et le rayonnement $\beta^{-}$ (électron négatif) a une énergie de $1,5 \mathrm{MeV}$ maximum. Le ${ }^{89} \mathrm{Y}$ est stable.

Aujourd'hui le ${ }^{89} \mathrm{Sr}$ est synthétisé dans les réacteurs nucléaires comme produit de fission de l'uranium-235 et/ou par absorption de neutrons par le ${ }^{88} \mathrm{Sr}$. C'est seulement après plusieurs décennies de chape de silence imposée sur tout ce qui concerne le nucléaire par les autorités politiques et militaires américaines, que les découvertes de Pecher de 1941 furent littéralement ressuscitées. L'idée d'utiliser le ${ }^{89} \mathrm{Sr}$ fut en effet redécouverte en 1974-1976 [4] par des chercheurs allemands et commercialisée aux Etats-Unis en 1993 sous le nom de Metastron, une solution de chlorure de strontium-89 injectable en intraveineuse. Les détails de la véritable saga historico-scientifique sont donnés dans la publication d'Evelyne [1]. L'effet palliatif (antalgique) du Metastron consiste, comme le démontra Pecher, dans l'irradiation interne localisée et spécifique des métastases osseuses causées, par exemple, par le cancer de la prostate ou du sein. En Belgique, le Metastron n'est plus utilisé et a été remplacé par le chlorure de radium-223 $\left({ }^{223} \mathrm{Ra}\right.$ ) (sous le nom commercial de Xofigo). Cet isotope du $\mathrm{Ra}$ qui, tout comme le $\mathrm{Sr}$ à faibles doses, est traité par l'organisme en analogue du $\mathrm{Ca}$, est un émetteur $\alpha\left(\mathrm{He}^{++}\right.$de 5,8 MeV) d'environ 11 jours de demi-vie. Le rayonnement $\alpha$ a une portée (fraction de $\mathrm{mm}$ ) beaucoup plus courte que celle (quelques $\mathrm{mm}$ ) du rayonnement $\beta^{-} \mathrm{du}^{89} \mathrm{Sr}$ dans les tissus organiques et est donc moins toxique pour les tissus sains autour des lésions.

Au printemps de 1941, Pecher reçoit l'ordre du gouvernement belge en exil à Londres [5] de rejoindre une force armée belge en reconstruction dont un contingent est à l'entrainement à Joliette, près de Montréal, Canada. Or en octobre 1940, en l'absence de toute armée belge dans une Belgique occupée, Pecher s'était enrôlé dans l'armée américaine. Sur la base d'une loi internationale, son "draft board" lui interdisait dorénavant de quitter les USA. Telle est l'origine du dilemme cornélien auquel fut confronté notre héros. Le dilemme enclencha une série de conseils, ordres et contrordres impliquant le gouvernement belge, la BAEF, des scientifiques de très haut niveau (dont Ernest Lawrence), des diplomates et jusqu'au $31^{\text {ème }}$ président des USA, Herbert Hoover, créateur, avec Sengier (voir ci-dessous), de la BAEF. Mais rien ne fit plier le gouvernement belge pourtant spécialement informé du grand intérêt pour les Alliés de maintenir Pecher au laboratoire de Berkeley. Celui-ci allait bientôt être entièrement dédié à l'effort de guerre, dans le cadre du projet Manhattan pour la construction de la bombe atomique initialement destinée à se prémunir d'un projet similaire en Allemagne nazie. Dans son obstination de ne faire aucune exception, le gouvernement belge, utilisant des méthodes de la guerre précédente, n'hésita pas à brandir la menace suprême de désertion pour faire respecter ses ordres et obliger Pecher à faire du drill et des marches forcées à Joliette. Les détails, insupportables, sont rapportés dans la référence [1]. Pecher fut complètement désemparé et jeté dans un état de confusion terrible. Malgré la perspective de sa paternité imminente (Evelyne allait naître quelques semaines après la tragique disparition) et son désir de servir l'armée belge dans sa capacité de médecin, il ne put résister à l'atroce angoisse qu'il tenta d'apaiser au moyen d'un puissant sédatif (barbiturique). Il fut retrouvé mort sur les bords de la rivière Assomption au nord de Joliette fin août 1941. Selon Evelyne Pecher [1] et les conclusions du médecin légiste («suicide par empoisonnement dans un moment d'aliénation 
mentale »), il s'agirait d'un suicide. L'hypothèse d'une "élimination", évoquée en raison de la proximité et la grande familiarité de Pecher avec tant de personnes informées des projets atomiques secrets à Berkeley, est sans réel fondement [1]. Reste la possibilité de l'accident. L'absence de témoin oculaire et de message sur les intentions de la victime laisseront à tout jamais planer un doute sur ce qui s'est réellement passé. Pecher rejoignait ainsi, comme Henry Moseley avant lui et Alan Turing après, la cohorte des jeunes et brillantes victimes expiatoires des guerres et des préjugés.

Ce qui est certain par contre, avec toute la considération due aux autorités belges de Londres, c'est leur manque de discernement dans cette affaire. Cet aveuglement est sans doute attribuable au fossé d'incompréhension séparant les deux cultures, l'humaniste et la scientifique, qu'allait dénoncer C.P. Snow [5] peu de temps après la guerre. Sans beaucoup de délicatesse, Snow qualifia les dirigeants politiques de son époque, pour la plupart des "humanistes" ou "littéraires", "d'analphabètes scientifiques". Leur état d'esprit s'inspirerait forcément, selon Snow, du principe que les sciences, du moins les sciences dites "dures" ou "exactes", constituent une culture mineure et que par conséquent, pour reprendre le dicton Churchillien, "scientists should be on tap, not on top". Churchill lui-même, affirmant l'exclusivité de ses responsabilités politiques, menaça Niels Bohr, le prix Nobel de 1922, père adulé et immense figure de la physique quantique moderne, d'emprisonnement pour avoir suggéré de partager les "secrets" atomiques avec Staline. Cet incident révélateur se produisit lors d'une audience accordée par Churchill à Bohr en mai 1944 à Londres au terme de laquelle Bohr fut poliment prié de prendre la porte. Le clairvoyant homme de science avait futilement tenté de prévenir le grand homme d'État de l'amorce d'une course aux armements nucléaires très dangereuse pour l'avenir de l'humanité. Outre Atlantique, le même principe fut appliqué sans ménagement lorsqu'en juillet 1945 le président Truman, ses collaborateurs et les militaires ne tinrent aucun compte de la pétition contre l'usage de la bombe atomique sur les villes japonaises, pétition signée, pour des raisons morales, par une centaine de scientifiques et techniciens, ceux-là même qui, sous la menace hitlérienne, avaient contribué à développer l'arme ultime. Toute proportion gardée, les recommandations de Lawrence (prix Nobel, 1939) en support de Charles Pecher n'eurent pas d'avantage d'effet sur les dirigeants politiques belges. Ceux-ci ont manifestement estimé, peut-être inconsciemment, que les scientifiques, quel que soit leur rang, n'ont rien à dire aux responsables chargés de la conduite des affaires d'État. Entretemps, le fossé entre les deux cultures s'est apparemment réduit quelque peu, par exemple lorsque certains gouvernements font appel à des "conseillers scientifiques". Pour autant les ténors de la culture humaniste sont encore loin d'envisager d'organiser démocratiquement le partage du pouvoir exécutif avec les ténors de la culture scientifique, quand bien même ce sont les créations de cette dernière, la "culture mineure", qui changent le cours des affaires humaines à une allure vertigineuse.

\section{Edgar Sengier.}

Edgar Sengier est né le 9 octobre 1879 à Courtrai. Il fréquente le Sint-Amands college de Kortrijk puis fait des études d'ingénieur des mines (1903) et d'ingénieur électricien (1904) à l'Université de Louvain. Sa carrière professionnelle [7] commence par un séjour en Angleterre, un stage en entreprise belge et une mission en Chine. En 1911 il rejoint l'UMHK. 
Son rôle comme membre puis comme dirigeant de cette firme (créée en 1906) est raconté dans la notice biographique très complète écrite par son collègue Edgar Van Der Straeten, lui aussi dirigeant de l'UMHK [8]. Nous n'y reviendrons pas ici. Examinons seulement ses activités liées à l'uranium et à la seconde guerre mondiale.

L'histoire des sciences du $20^{\text {ème }}$ siècle aboutissant à la libération de l'énergie nucléaire à grande échelle est plutôt discrète sur l'approvisionnement primaire en uranium. Par exemple dans son récit épique de près de 900 pages sur cette épopée humaine à la fois prodigieuse et tragique, l'écrivain américain Richard Rhodes [9] réserve peu d'espace à la fourniture initiale du minerai d'uranium au projet Manhattan pour la construction de la bombe. Ni l'index ni la bibliographie de cette œuvre magnifique et définitive ne mentionnent le nom d'Edgar Sengier, le pourvoyeur de l'ombre de l'indispensable matière première.

Cette discrétion s'explique par plusieurs raisons. Lorsqu'en 1942 l'officier américain tout juste mis en charge du projet Manhattan, le général Leslie Groves [10], eut sérieusement à se préoccuper d'acquérir l'uranium pour le projet, quelle ne fut pas sa surprise et sa satisfaction d'apprendre que ce problème avait été résolu auparavant. Grâce à l'initiative de Sengier, des quantités de riche minerai plus que suffisantes avaient déjà été entreposées à Staten Island, N.Y., deux ans avant que la fabrication de la bombe ne fut ordonnée par le président Roosevelt. Groves disposait donc de l'uranium dont il avait besoin, d'autant que Sengier était à disposition pour obtenir des quantités supplémentaires pratiquement illimitées du minerai. Le problème d'acquisition de l'uranium réglé, plus la peine d'en parler ! La seconde raison est l'obsession de Groves pour le secret militaire [9], [10] dont nous parlions plus haut à propos de l'œuvre de Pecher. Pendant et après la seconde guerre mondiale, la nervosité anticommuniste des militaires et politiques américains imposa le secret absolu sur toute information de type nucléaire, en particulier sur les détails concernant les sources de minerai. Ainsi le rôle singulier de Sengier dans l'avènement du nucléaire militaire et industriel au milieu du $20^{\text {ème }}$ siècle ne fut révélé publiquement qu'entre sa retraite en 1960 et son décès en 1963, période pendant laquelle les Américains relaxèrent quelque peu leur régime du secret militaire. C'est aussi à ce moment que Groves [10] publie ses mémoires de guerre dans lesquels il rend hommage à Sengier. Il y raconte, on ne peut plus officiellement, le rôle définitif joué par l'homme d'affaires dans la réussite du projet Manhattan. Enfin, Sengier fit lui-même preuve d'une discrétion absolue dans son engagement aux côtés des Alliés [11].

Pour le présent récit nous retiendrons quelques dates clé sur les actions personnelles de l'homme d'affaires :

1914. Sengier est envoyé à Londres pour diriger les services de l'UMHK qui fournira la totalité de sa production aux alliés pendant la première guerre mondiale.

1917. Sengier est chargé à Londres de la trésorerie de la "Hoover Commission for Relief in Belgium" (CRB), une aide en nourriture et une assistance au relèvement économique et industriel accordées par les USA à la Belgique occupée par les Allemands. Le président de cette commission, l'américain Herbert Hoover, un ingénieur des mines comme Sengier, deviendra le $31^{\text {ème }}$ président des USA (1929-1933). Dans la commission, Sengier travaillera et se liera d'amitié avec Émile Francqui. Après la guerre, grâce au reliquat des fonds de la Commission, ces trois responsables de la CRB seront à la base de la création rien moins que 
la Fondation Universitaire (1920), la BAEF (1920), le FNRS (1928) et la Fondation Francqui (1932). L'auteur du présent article et nombreux de ses lecteurs et auditeurs ont bénéficié ou bénéficient encore de l'aide d'une ou de plusieurs de ces Fondations. Naturellement, Sengier restera un interlocuteur privilégié de Hoover lequel, comme on l'a dit, sera en première ligne dans l'intervention, malheureusement futile, en faveur de Charles Pecher, alumnus de la BAEF.

1918. Sengier prend l'initiative prémonitoire de faire activer les travaux sur les riches minerais d'uranium découverts en 1913 au Katanga. Il incite l'UMHK à en faire entreprendre le traitement par la Société Générale Métallurgique d'Hoboken/Olen dont Sengier est aussi l'Administrateur-Délégué. Des milliers de tonnes de pechblende (le minerai d'uranium), "déchet" de l'extraction du radium (réalisée pour la première fois par le couple Marie et Pierre Curie), commencent à s'accumuler à Shinkolobwe (Katanga) et en Belgique.

1939. A New York où il s'est réfugié sous la menace allemande, Sengier crée la filiale de l'UMHK, African Metals Corporation (Afrimet), d'où il organise la fourniture aux Alliés des minerais de métaux stratégiques congolais $(\mathrm{Cu}, \mathrm{Co}, \mathrm{Sn}, \ldots)$ pour la seconde guerre mondiale.

En ce qui concerne spécifiquement l'uranium, avant même de quitter la Belgique, Sengier avait déjà pris sur lui de faire évacuer les réserves de pechblende présentes sur le sol belge, en vue de soustraire ces ressources aux Allemands. Ensuite, depuis Afrimet, il fait expédier directement du Katanga à New York 1.200 tonnes du minerai qui arriveront fin 1940 et seront stockés dans le Port de Richmond sur Staten Island. Ni les autres dirigeants de l'UMHK, ni le premier ministre belge, ni le ministre des colonies, ni l'ambassadeur de Belgique à Washington ne sont informés de ce transport discret vers les États-Unis. «J'ai fait cela en ne disant rien ni à personne » confiera-t-il après la guerre [11]. Telles étaient sa singulière autorité et son indépendance d'action !

L'initiative de déménager son quartier général à N.Y. est facilement compréhensible en raison du danger que Sengier a dû pressentir de devenir, avec sa firme, l'otage des Allemands. Mais pour l'uranium il avait une raison supplémentaire d'agir et de le faire vite. Car déjà en mai 1939, le magnat mondial du radium qu'il était devenu depuis 1918, en possession d'une montagne de pechblende "résiduelle", avait été mis personnellement et secrètement "au parfum nucléaire" par Fréderic Joliot-Curie à Paris et Henry Tizard (recteur de l'Imperial College) à Londres. Ces deux éminences scientifiques voulaient s'assurer, au nom de leurs pays respectifs, de l'acquisition exclusive de cette matière soudainement devenue hautement convoitée. Alerté et informé confidentiellement par ces deux sources indépendantes et fiables, Sengier a dû prendre conscience de l'énorme intérêt stratégique que revêtait la pechblende pour les affaires de l'UMHK et pour l'avenir industriel et commercial de la Belgique et de sa colonie. Encore fallait-il qu'il fût doté d'une intuition étonnante pour être capable de démêler le réel de la fiction dans les promesses exagérées et les visions futuristes que lui faisaient miroiter les deux éminents spécialistes. Après tout, en mai 1939, la découverte de la fission datait seulement de quelques mois et jusque-là le phénomène n'était qu'une observation sur une simple table de laboratoire. De plus, une réaction de fission en chaîne dans l'uranium pour libérer l'énergie nucléaire à grande échelle n'était encore qu'une perspective incertaine pour le futur plus ou moins lointain. Sengier promit quelques centaines de tonnes du matériau aux 
Français. Quant aux Britanniques, Tizard essuya un refus à sa demande de fourniture exclusive de l'uranium congolais. Avant de se quitter, il conseilla Sengier en ces termes : "Monsieur, réfléchissez bien. Si les matières d'Urane dont vous disposez devaient jamais tomber dans les mains d'un ennemi possible, ce serait pour nos pays une catastrophe nationale. Quoi que vous fassiez, mettez ces matières en sécurité et surtout faites-le dans le plus grand secret". Un conseil qui, manifestement, ne tomba pas dans l'oreille d'un sourd!

C'est en janvier 1942, juste après Pearl Harbor, que le président Roosevelt ordonne formellement de faire construire la bombe atomique [9]. Une nouvelle équipe, essentiellement militaire cette fois, est formée par le Corps du Génie de l'armée américaine, le MED (Manhattan Engineering District) ou brièvement Manhattan. La question de la fourniture d'uranium se pose maintenant avec urgence. Plusieurs mois s'écoulent encore avant que, le 17 septembre, le général Leslie Groves ne soit placé à la tête du projet [10]. Et dès le lendemain, Groves envoie son aide, le colonel Kenneth Nichols, interroger Sengier dans ses bureaux d'Afrimet. Entretemps, le dépôt de Staten Island avait été pratiquement "oublié". Après que l'homme d'affaires eut vérifié les accréditations de son visiteur, la conversation surréaliste suivante intervint entre les deux hommes :

Nichols: Nice to meet you. Comment vous sentez-vous ici aux Etats Unis ?

Sengier: Très bien, merci. Mais, dites-moi Colonel, êtes-vous ici pour bavarder ou pour parler affaires?

Nichols: Parler affaires. Pourriez-vous aider à fournir à l'Amérique du minerai d'uranium du Congo belge? C'est vital pour la cause alliée.

Sengier: Quand vous le faut-il?

Nichols: Si cela n'était pas impossible, je dirais demain.

Sengier: Ce n'est pas impossible. En ce moment même, le minerai est ici à New York. Mille tonnes de minerai. J'attendais votre visite depuis longtemps!

Quelques instants après cette conversation hallucinante, Nichols, planant comme sur un nuage, sort des bureaux de Sengier avec un bout de texte manuscrit signé par l'homme d'affaires et déclarant que le stock de Staten Island est maintenant propriété des Etats-Unis, selon un accord et pour un prix à convenir plus tard ! Le tout sans la moindre consultation d'autres dirigeants de l'UMHK (maintenant hors d'atteinte dans la Belgique occupée), ni du gouvernement belge en exil (qui restera, jusqu'en avril 1944, dans l'ignorance totale des affaires nucléaires qui se trament entre les Alliés). On comprend pourquoi, à partir de cette transaction incroyable, Sengier déjà très proche de l'administration gouvernementale précédente et de l'ancien président Hoover lui-même [8], jouira dorénavant d'un traitement royal dans toutes ses interactions avec les plus hautes autorités de son pays hôte. Il en eut probablement été de même à l'égard de Pecher si l'ampleur de ses contributions à la science et de ses potentialités pour la cause alliée, n'avaient été incomprises ou ignorées par le gouvernement belge en exil.

En 1944, le volume des affaires menées confidentiellement et secrètement avec la seule personne de Sengier ne cessant d'augmenter, les Alliés réalisent la nécessité d'approcher le gouvernement belge. Les Anglo-Américains veulent acquérir les droits exclusifs sur tout 
l'uranium restant encore dans les mines congolaises. Sengier est convoqué d'urgence à Londres le 16 avril 1944 par le gouvernement belge pour l'informer et l'assister dans les négociations. Les Américains se félicitent de ce choix car ils sont en excellents termes avec l'homme d'affaires depuis des décennies et spécialement suite à l'apport providentiel de Staten Island et d'autres fournitures pendant la guerre. On peut supputer que, confrontés à un refus ou à une attitude récalcitrante du gouvernement belge, les Anglo-Américains auraient pu, en l'absence de notre héros, confisquer purement et simplement le matériau nucléaire stratégique (comme ils ne manquèrent pas de le faire avec les minerais inutilisés par le projet nucléaire avorté de l'Allemagne en ruine). L'élaboration d'un plan détaillé sera poursuivie par trois hommes : Sengier à New York pour Afrimet et la Belgique, Groves pour les Américains et Sir Charles Hombro pour les Britanniques. Un Accord Tripartite fut finalement paraphé par le gouvernement belge le 5 septembre 1944, quelques jours avant son retour à Bruxelles libérée, le 8 septembre. Le 20 septembre, le ministre des affaires étrangères, Paul Henri Spaak, expliquera à l'ensemble du gouvernement réuni à Bruxelles, en séance secrète, l'essentiel de la convention uranium avec les Alliés et ratifiera l'Accord Tripartite. Cet Accord comporte dix articles. L'article 9, le plus important pour le développement à long terme du nucléaire civil en Belgique [11], concerne les conditions d'utilisation des minerais à des fins industrielles de production d'énergie. Par cet article les Américains et Britanniques acceptent la participation de la Belgique à cette utilisation en "des termes équitables", sans plus de précision. Enfin l'article 10 impose que la convention uranium soit traitée comme un secret militaire, en conformité avec l'objectif principal de l'Accord (selon l'article 1, rien moins que "protéger la civilisation", de la menace communiste on suppose).

Le jour d'Hiroshima, le 6 août 1945, Sengier est invité par Groves à écouter la radio annonçant le premier bombardement nucléaire de l'histoire. Personne ne sait quelle fut la réaction intime de l'homme d'affaires. Trois jours plus tard, quand Nagasaki à son tour est oblitérée, Sengier se trouve alors à Washington où il est l'invité de Groves. Celui-ci l'introduit au Président Truman à la Maison Blanche en ces termes: "Je vous présente l'homme sans l'assistance duquel nous n'aurions pu accomplir ce que nous avons fait". Le lendemain, Sengier écrit une lettre à Groves pour le féliciter de "cette invention remarquable ... qui aura non seulement des répercussions immédiates sur la poursuite de la guerre mais aussi sur le sort du monde et de l'humanité". Pour la poursuite et la fin de la guerre il n'y avait plus que quelques heures à patienter. Mais pour le sort du monde, l'humanité patiente toujours et est condamnée, semble-t-il, à attendre indéfiniment dans le cadre inconfortable et fragile de la stratégie MAD (Mutual Assured Destruction) et du Traité de Non-prolifération (NPT) des armes nucléaires. Après la guerre, pour l'UMHK une nouvelle décennie d'expansion extraordinaire commençait, sous l'impulsion de Sengier. Enfin, grâce à la vigilance de Sengier et des signataires belges de l'Accord Tripartite, une aventure scientifique, technique et industrielle s'ouvrait dans le domaine du nucléaire civil pour la Belgique [12].

Le 19 avril 1946 Sengier retourne aux États-Unis, convoqué par Groves pour lui remettre la Medal of Merit, la plus haute distinction décernée à un civil américain ou étranger, signé par le Président Truman, pour "Fidélité extraordinaire et conduite exceptionnellement méritoire". Cette décoration et quelques autres [8] couronnaient la carrière d'un homme d'exception. On 
se prend à rêver sur ce qu'aurait été le parcours de Charles Pecher s'il lui avait été permis de continuer à déployer ses multiples et immenses talents.

\section{Remerciements.}

Je remercie le prof. Guy Maghuin-Rogister, Secrétaire Général de la SRSL, de m'avoir invité à prononcer le discours de clôture de cette réunion 2018 de la SRSL consacrée à la Radioactivité. Je salue la présence d'Evelyne Cerf-Pecher à cette réunion et la remercie de m'avoir confié son livre très fouillé sur la biographie et l'œuvre scientifique de son père. Je suis redevable aussi au prof. Frank Deconinck, Professor Em. Vrije Universiteit Brussel, pour m'avoir aidé de son expertise sur Charles Pecher. Enfin, je remercie le prof. François Jamar, Chef du Service de Médecine Nucléaire des Cliniques Universitaires Saint-Luc, pour de précieux renseignements sur des radioéléments médicaux.

\section{Références.}

[1] Evelyne Cerf-Pecher, Charles Pecher et le Strontium Radioactif, édition remaniée et complétée du livre de cet auteur intitulé Charles Pecher - L'homme de Sciences-1913-1941, Didier Devillez, Editeur, ISBN 978-2-87396-132-9 (2011).

[2] André Jaumotte et Frank Deconinck, Nouvelle Biographie Nationale 13, Académie Royale de Belgique, 226-269 (2016).

[3] Charles Pecher (posthume), Biological investigations with radioactive calcium and strontium. Preliminary report on the use of radioactive strontium in the treatment of metastatic bone cancer, Univ. Cal. Pub. Pharmacol. 2, 117-150 (1942).

[4] https://www.sciencedirect.com/science/article/pii/S0022534717590023

[5] La formation et la composition (changeante) de ce gouvernement en exil font l'objet d'un mémoire fouillé de l'historien Jean Stengers, Sur l'histoire du gouvernement belge de Londres, Revue Belge de Philosophie et d'Histoire, 78-3-4, pp. 1009-1022.

[6] Charles, Percy Snow, The two Cultures, Cambridge UP (1959), ISBN 0-521-45730-0. https://en.wikipedia.org/wiki/The_Two_Cultures

[7] Émile Fossoul, dans Témoignages, Un demi-siècle de nucléaire en Belgique, Presses Universitaires Européennes, Bruxelles, p.55, 1994.

[8] Edgar Van der Straeten, Sengier (Edgar), Académie Royale des Sciences d'Outre-Mer, Biographie Belge d'Outre-Mer T. VII-A, col. 429-437 (1973).

[9] Richard Rhodes, The Making of the Atomic Bomb, Touchstone Book, 1986. (prix Pulitzer).

[10] Leslie R. Groves, Now it can be told, Harper and Row, 1962. Groves, le chef militaire du projet Manhattan, consacre un chapitre de 4 pages au problème de l'approvisionnement en minerai d'uranium. 
[11] Dans son article du Reader's Digest de décembre 1953 intitulé The Mystery Man of the ABomb, le journaliste John Ghunter souligne l'effacement et la discrétion que Sengier s'est imposés dans toutes ses activités liées au nucléaire pendant et après la guerre. Cette discrétion, naturelle chez un homme d'affaires et fortement conseillée par Tizard, fut d'ailleurs promptement convertie en obligation de secret absolu par Groves qui, en outre, veilla à flanquer Sengier de deux "anges gardiens" dans tous ses déplacements.

[12] A. Jaumotte, Le début du nucléaire en Belgique, dans Bulletin de la Classe des Sciences. Académie Royale de Belgique, t. 14, 7-12, p. 245-254 (2003). 